\title{
Effective Use of Linear DNA in Cell-Free Expression Systems
}

\author{
Megan A. McSweeney and Mark P. Styczynski * \\ Georgia Institute of Technology, School of Chemical \& Biomolecular Engineering, Atlanta, GA, United States
}

Cell-free expression systems (CFEs) are cutting-edge research tools used in the investigation of biological phenomena and the engineering of novel biotechnologies. While CFEs have many benefits over in vivo protein synthesis, one particularly significant advantage is that CFEs allow for gene expression from both plasmid DNA and linear expression templates (LETs). This is an important and impactful advantage because functional LETs can be efficiently synthesized in vitro in a few hours without transformation and cloning, thus expediting genetic circuit prototyping and allowing expression of toxic genes that would be difficult to clone through standard approaches. However, native nucleases present in the crude bacterial lysate (the basis for the most affordable form of CFEs) quickly degrade LETs and limit expression yield. Motivated by the significant benefits of

OPEN ACCESS

Edited by:

Yong-Chan Kwon,

Louisiana State University,

United States

Reviewed by:

Cheemeng Tan,

University of California, Davis,

United States

Nigel Reuel,

lowa State University, United States

*Correspondence:

Mark P. Styczynski

mark.styczynski@chbe.gatech.edu

Specialty section:

This article was submitted to

Synthetic Biology,

a section of the journal

Frontiers in Bioengineering and

Biotechnology

Received: 26 May 2021

Accepted: 06 July 2021

Published: 20 July 2021

Citation:

McSweeney MA and Styczynski MP (2021) Effective Use of Linear DNA in Cell-Free Expression Systems.

Front. Bioeng. Biotechnol. 9:715328.

doi: 10.3389/fbioe.2021.715328 using LETs in lieu of plasmid templates, numerous methods to enhance their stability in lysate-based CFEs have been developed. This review describes approaches to LET stabilization used in CFEs, summarizes the advancements that have come from using LETs with these methods, and identifies future applications and development goals that are likely to be impactful to the field. Collectively, continued improvement of LET-based expression and other linear DNA tools in CFEs will help drive scientific discovery and enable a wide range of applications, from diagnostics to synthetic biology research tools.

Keywords: cell-free expression, linear expression template, nuclease inhibition, genetic circuits, rapid prototyping, DNA aptamers

\section{INTRODUCTION}

Cell-free expression systems (CFEs) are powerful tools for the execution of arbitrary genetic programs or the synthesis of proteins in vitro. One of the most common and affordable forms of CFE, the lysate-based system, is composed of a crude cellular extract (typically from E. coli, but lysates from other organisms are useful for specific applications) combined with supplemented cofactors and substrates essential for transcription and translation. CFEs offer several advantages over the use of whole-cell in vivo systems (Khambhati et al., 2019). Transport limitations inherent to whole-cell systems due to the cell membrane are reduced in CFEs because they have no membrane, yielding improved control over plasmid dosage, $\mathrm{pH}$, and inducer levels (Swartz 2006). CFEs also eliminate cellular toxicity issues that sometimes arise in vivo from the expression of certain proteins, with the additional effect of avoiding plasmid instability often caused by toxicity (Katzen et al., 2005). CFEs have expedited research on key biological principles (Nirenberg and Leder 1964) and have been applied in contexts ranging from industrially relevant large-scale protein production (Zawada et al., 2011) to upstream screening for glycoprotein synthesis (Schoborg et al., 2018). CFEs are particularly 


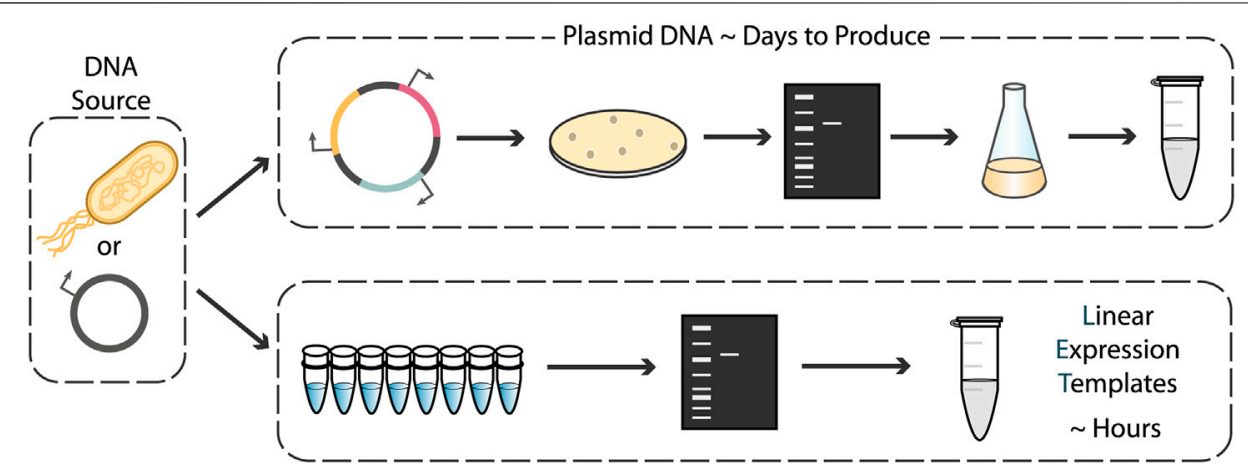

FIGURE 1 | Strategies for preparing DNA templates for use in cell-free expression systems. Traditional plasmid cloning protocols involving construct assembly, transformation, screening, and plasmid purification take days to complete. Linear expression templates can be made via PCR from genomic DNA or plasmid templates and used directly after amplicon verification and purification, drastically reducing DNA template preparation time.

attractive for sensor development, with applications ranging from environmental sensors (Verosloff et al., 2019; Thavarajah et al., 2020) to user-friendly biomedical diagnostics (McNerney et al., 2019).

The configuration of CFEs as a membrane-less solution of expression machinery, compared to the membranecompartmentalized individual cells of in vivo systems, not only reduces the transport limitations that would have been imposed by the membrane, but also makes the use of linear expression templates (LETs) a viable option for CFEs. For in vivo systems, the use of plasmids is required for stable expression without genomic integration. Since delivery of DNA into cells happens with low efficiency, successfully transformed cells must be selected for and then expanded, meaning that the DNA vector must replicate during cell growth to avoid growth-associated dilution and loss. Plasmid construction requires cloning, in vivo synthesis, and plasmid isolation, a process that takes days for each new construct. However, LETs - which typically consist of a promoter region, gene coding sequence, and transcriptional terminator-can be quickly and easily produced in vitro via polymerase chain reaction (PCR) from existing plasmid DNA or genomic DNA (Figure 1). With techniques such as Golden Gate assembly, multiple LETs can be rapidly assembled into complex constructs entirely in vitro. Using PCR products as expression templates rather than plasmids can decrease the "primers-to-testable-DNA" time from days to only a few hours. This can facilitate high-throughput screening and significantly accelerate the prototyping cycle time of multicomponent genetic circuits to a standard business day (Sun et al., 2013).

Additionally, LETs allow for expression of toxic genes in CFEs that otherwise would be difficult to clone into a plasmid. The LET construct for the toxic gene can be amplified directly from genomic DNA or from a plasmid without a promoter upstream of the gene. Without a promoter, the plasmid containing the toxic gene can be cloned because the gene will not be expressed in vivo as normal. This can then be used as a template for a LET, with the promoter sequence added via primers during PCR.
Despite the numerous benefits of LETs, plasmids remain the most widely used DNA template in CFEs due to their resistance to degradation. DNA nucleases native to $E$. coli are present in the crude cellular lysate and remain after lysate purification. These nucleases readily digest linear double-stranded (ds) or singlestranded (ss) DNA fragments in the reaction to an extent not observed for circular plasmids, causing LETs to have a much shorter half-life than plasmids. This leads to much lower protein yields or diminished function of genetic circuits, which has in turn slowed adoption of LETs for CFEs.

However, there exist several approaches for stabilizing linear DNA that lessen the impacts of these phenomena and allow for the effective use of LETs in crude lysate-based CFEs. In this review, we will describe these advances and compare their effectiveness and limitations. Then, we will summarize select applications where LETs have been used to expedite circuit prototyping cycles, rapidly screen synthetic regulators, express toxic proteins, and more. Lastly, we will discuss the restrictions of the existing nuclease inhibition strategies, recognize areas with critical need for improvement, and identify future development goals.

\section{APPROACHES FOR STABILIZING LINEAR DNA IN CELL-FREE EXPRESSION SYSTEMS}

While crude bacterial lysate is widely used for CFEs, purified recombinant proteins can also constitute the basis for CFEs. PURExpress (NEB), PUREfrex 2.0, and Magic PURE are three commercially available recombinant systems based on the original PURE system developed by Shimizu et al. (2001) (See also Laohakunakom et al. (2020)). Purchasing these systems from commercial vendors saves valuable laboratory time compared to typical modern protocols for in-house preparation of crude cell extract that typically take three days and over $10 \mathrm{~h}$ of active labor (Kwon and Jewett 2015). Perhaps more importantly, these systems have minimal nuclease and protease activity compared to crude lysates, making expression from LETs much easier than in lysates. 

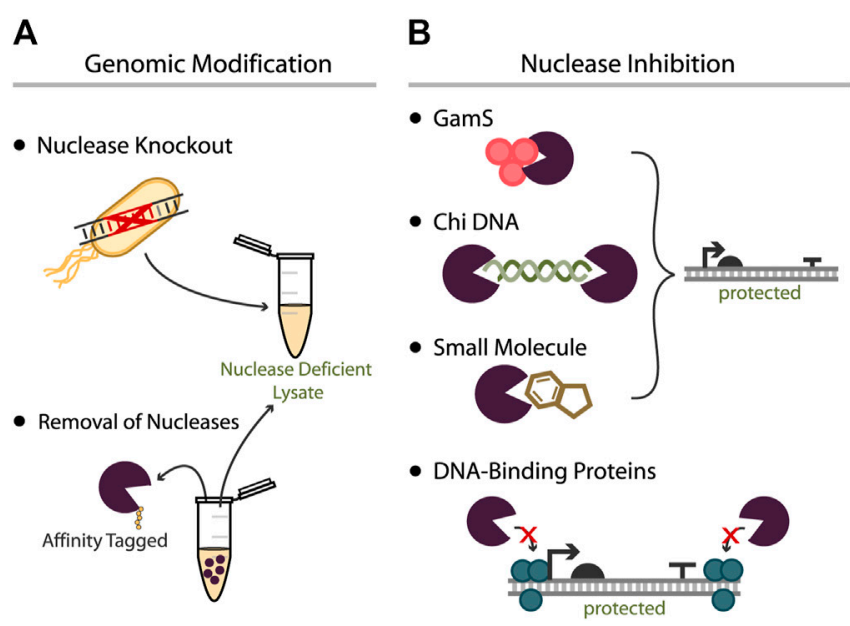

\section{C LET Modifications}

- DNA Methylation

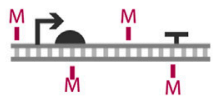

- Phosphorothioate Linkages

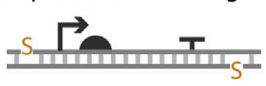

- In vitro circularization

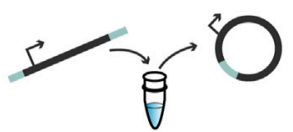

FIGURE 2 | Approaches used to increase expression yield from LETs. (A) Bacteria can be genetically modified prior to lysate extraction either by deletion of nuclease genes or by the fusion of affinity peptides to nuclease genes for later removal during lysate processing. Both methods aim to produce a lysate with negligible nuclease activity. (B) Nuclease inhibitors can be added to cell-free reactions to mitigate activity of specific nucleases. This effect can be achieved with direct RecBCD inhibitors such as GamS, Chi DNA, and certain small molecules, or via the addition of DNA-binding proteins that interact with the LETs. (C) The LET can also be modified to better protect the construct from nuclease degradation: methylation of PCR-generated LETs can mimic the chemistry of native DNA, phosphorothioate linkages can be added to the ends of LETs via modified primers, or amplicons with appropriately designed primers can be recircularized prior to CFE.

TABLE 1 | Effectiveness of different nuclease inhibition strategies quantified by their ability to improve LET-based expression in E. coli CFEs. Values with an asterisk were inferred from figures in the corresponding reference.

\begin{tabular}{|c|c|c|c|c|}
\hline \multicolumn{2}{|c|}{ LET stabilization approach } & \multirow{2}{*}{$\begin{array}{c}\text { Improvement } \\
3-6 x\end{array}$} & \multirow{2}{*}{$\begin{array}{c}\text { Metric } \\
\text { Fold change from WT strain }\end{array}$} & \multirow{2}{*}{$\begin{array}{l}\text { References } \\
\text { Michel-Reydellet } \\
\text { et al. (2005) }\end{array}$} \\
\hline $\begin{array}{l}\text { Genomic } \\
\text { modifications }\end{array}$ & $\Delta$ recCBD::Plac-red-kan- $\Delta$ endA & & & \\
\hline & Affinity tag removal of RecD and PNPase & $4 x$ & Fold change from WT strain & Seki et al. (2009) \\
\hline \multirow[t]{5}{*}{ Nuclease inhibition } & GamS & $37.6 \%$ & $\begin{array}{l}\text { Percentage of plasmid } \\
\text { expression }\end{array}$ & Sun et al. (2013) \\
\hline & Chi DNA & $23 \%^{*}$ & $\begin{array}{l}\text { Percentage of plasmid } \\
\text { expression }\end{array}$ & Marshall et al. (2017) \\
\hline & Small molecule RecBCD inhibitors & $\begin{array}{c}\text { 250\%* (CID 697851) } \\
300 \% *(C I D ~ 1517823)\end{array}$ & $\begin{array}{l}\text { Percent increase from no } \\
\text { inhibitor }\end{array}$ & $\begin{array}{l}\text { Shrestha et al. } \\
\text { (2014) }\end{array}$ \\
\hline & ssCro & $23 \%$ & $\begin{array}{l}\text { Percentage of plasmid } \\
\text { expression }\end{array}$ & Zhu et al. (2020) \\
\hline & $\mathrm{Ku}$ & $8 \% *$ & $\begin{array}{l}\text { Percentage of plasmid } \\
\text { expression }\end{array}$ & Yim et al. (2020) \\
\hline \multirow[t]{3}{*}{$\begin{array}{l}\text { LET modifications } \\
\text { and enhanced design }\end{array}$} & DNA methylation & $\begin{array}{l}32 \% \text { (dam methyltransferase) } \\
-18 \% \text { (CpG methyltransferase) }\end{array}$ & $\begin{array}{l}\text { Percent increase from } \\
\text { unmethylated LET }\end{array}$ & Zhu et al. (2020) \\
\hline & Terminal phosphorothioate (PT) linkages (x2) & $36 \%^{*}$ & $\begin{array}{l}\text { Percent increase from } \\
\text { unmodified LET }\end{array}$ & Sun et al. (2013) \\
\hline & 3'-tail mRNA secondary structures & $\begin{array}{c}\text { 92\%* (poly }(G) \text { tail) } \\
\text { 265\%* (T7 terminator) }\end{array}$ & $\begin{array}{l}\text { Percent increase from LETs } \\
\text { lacking } 3^{\prime} \text { secondary structures }\end{array}$ & Ahn et al. (2005) \\
\hline
\end{tabular}

However, crude lysate-based CFEs still dominate the field based in part on their affordability. For example, the PURExpress in vitro protein synthesis kit costs $\$ 0.35-\$ 0.65$ per $1 \mathrm{uL}$ reaction while a crude lysate-based system costs $\$ 0.02-\$ 0.04$ per $1 \mathrm{uL}$ reaction (Pardee et al., 2014). Recently, robust methods have been reported for in-house production of recombinant protein CFEs by coculturing and inducing expression of all 36 proteins present in the commercial systems (Lavickova and Maerkl 2019). This "OnePot" method can achieve high protein yields at a cost closer to $\$ 0.09$ per $\mu \mathrm{L}$. While this is significantly lower than the cost of the PURExpress system, it is still more expensive than crude lysate CFEs and laborious in its own way; it remains to be seen whether this approach will be adopted widely.

The financial benefits of lysate-based CFEs, among other advantages, have motivated substantial effort toward addressing one of its major shortcomings: the effects of nucleases in crude extracts. Significant linear DNA degradation is attributed to exonuclease $\mathrm{V}$, the product of the $r e c B C D$ operon. As a result, previous efforts have attempted to remove, inhibit, or deter RecBCD activity on LETs (Figure 2), with varying success. The relative effectiveness of each approach is listed in Table 1. The most common approaches to achieve 
these goals include genomic removal of nucleases, nuclease inhibition, and protective linear DNA modifications.

\section{Genomic Modifications}

In $E$. coli, endonucleases and exonucleases are critical for proper cell function and are essential enzymes involved in doublestranded break repair and recombination events. The predominant endonuclease in $E$. coli is endonuclease I, encoded by the endA gene. The dominant source of exonuclease activity in $E$. coli comes from exonuclease $\mathrm{V}$, the product of the recBCD operon (Kuzminov and Stahl 1997). In this complex, RecB has both helicase and nuclease activity, and RecD serves only as a helicase. Attempts have been made to engineer E. coli mutants that lack the activity of one or several of these subunits (Figure 2A). For example, a cell extract made from an A19 $\Delta r e c D \Delta e n d A$ mutant was used for cell-free protein synthesis and tested with LETs for nuclease activity (Michel-Reydellet et al., 2005). Plasmid-based expression was slightly lower in the mutant extract compared to the wild type, suggesting that the endA deletion did not improve plasmid stability. However, extracts from both strains possessed significant linear DNA degradation as evidenced by low protein yields from the LETs. And while these results showed almost equal expression from plasmids between strains, others have reported that extracts of exonuclease-deficient strains exhibit significant loss in translational activity (Ahn et al., 2005).

Knockout strains that lack the entire $\operatorname{rec} B C D$ operon have also been explored. Complete recBCD knockouts create strains that are extremely slow-growing, cannot support recombination, and do not allow for replication of many plasmids (Yu et al., 2000). To address this, $r e c B C D$ can be replaced with the Red recombination system from bacteriophage $\lambda$ to remove exonuclease activity while allowing recombination and adequate growth rates (Murphy 1991; Michel-Reydellet et al., 2005). Lysates made from this mutant yielded over three times more protein from PCRgenerated LETs compared to the wild-type extract.

Since the growth defects of these knockout strains make the culture steps in lysate preparation more time-consuming, removing nucleases from the lysate post-harvest has been explored as an alternative option. Insertion of streptavidinbinding peptide tag sequences in the 3' termini of RecD and the gene encoding polynucleotide phosphorylase (PNPase) was shown not to effect $E$. coli growth (Seki et al., 2009). Lysates made from these modified strains were treated with an affinity purification resin to remove tagged proteins, leading to approximately four times as much GFP production from LETs compared to wild-type BL21 (Seki et al., 2009). Expanded to multiple other nucleases simultaneously, this approach could potentially have a substantial impact on linear DNA stability. That being said, genes that overlap on the chromosome cannot be tagged (and thus, removed), and physical limitations of the resin such as its binding capacity and specificity can limit the success of this approach. Additionally, the resin-treated lysate with the greatest improvement in LET protein yield exhibited a $15 \%$ loss in plasmid-driven protein expression, which reduces the overall potential yield of the system.

\section{Nuclease Inhibition}

Inhibiting nucleases present in the lysate instead of removing them completely is another promising approach to improving expression from LETs (Figure 2B). This strategy is potentially simpler and more easily generalizable than genetic modifications, since the addition of supplements to CFEs can be applied to lysates of any strain background. Multiple strategies for nuclease inhibition have been reported.

One widely used method for nuclease inhibition is inclusion of the bacteriophage $\lambda$ protein GamS in CFEs. GamS is an inhibitor of RecBCD that can protect linear DNA from degradation in vivo and in crude extracts (Murphy 1991; Sitaraman et al., 2004). GamS has been used in CFEs to allow expression from LETs containing various promoters, including T7, natural and synthetic $\sigma^{70}$, and well-characterized inducible promoters (Sun et al., 2013). With GamS supplementation, LETs with a strong $\sigma^{70}$ promoter yielded $37.6 \%$ of that from a plasmid, a large improvement from the $2 \%$ LET yield in the absence of GamS. The GamS protein can be purified with affinity tagging or purchased commercially specifically for use in CFEs. Enhanced extracts can also be made from strains that express GamS to improve LET performance without the need to exogenously add protein. These GamS-containing extracts doubled LET protein yield compared to extracts without GamS (Contreras-Llano et al., 2020). While this method is more cost-effective and avoids any negative effects caused by the protein storage buffer, the observed benefits in LET performance are far less than those seen when purified GamS is added. Additionally, enriched lysates made from plasmid-carrying strains can suffer from overall decreases in efficiency, a consequence of the burdens of plasmid maintenance (Zawada and Swartz 2006).

The use of Chi DNA for RecBCD inhibition also avoids the costly purchase or reagent-intensive purification of proteins. Chi DNA are dsDNA oligos containing the short DNA motif (5'GCTGGTGG-3') referred to as a Chi (crossover hotspot instigator) site. These sites can be found throughout the genome of $E$. coli and are known to be involved in recombination events. Chi sites act as recognition sequences for RecBCD and other proteins involved in recombination (Smith et al., 1981). Addition of dsDNA composed of six repeated Chi sequences (with spacers) to the cell-free reaction has been shown to stabilize LETs and improve protein yield from undetectable levels to approximately $23 \%$ of that from a plasmid (Marshall et al., 2017).

RecBCD activity can also be inhibited by organic small molecules. From an in vivo screen of 326,100 organic small molecules, several compounds were identified to inhibit the nuclease activity of RecBCD and AddAB, a RecBCD analog native to $B$. subtilis and other bacteria (Amundsen et al., 2012). Many of these compounds showed reduced helicase and Chi-cutting activity from RecBCD as well. Two of these small molecules, CID 697851 and CID 1517823, improved expression from LETs in CFEs as much as $200 \%$ at some concentrations compared to reactions with no RecBCD inhibitors (Shrestha et al., 2014). However, expression remained far less than that from a plasmid. 
GamS, Chi DNA, and small molecule RecBCD inhibitors all function by binding directly to RecBCD, but nuclease inhibitors can also bind directly to the LET to protect it from degradation. For example, adding single-chain Cro (ssCro) to a cell-free reaction protects LETs containing the ssCro operator recognition sequences from RecBCD degradation (Jana et al., 1998; Zhu et al., 2020). These ssCro operators can be easily added to LETs through PCR. Addition of ssCro improved LET expression over 6-fold, resulting in about $23 \%$ of plasmid-based expression (Zhu et al., 2020). DNA-binding proteins can also inhibit degradation from other exonucleases. $\mathrm{Ku}$, for example, is a DNA-binding protein with homologs found in eukaryotes and prokaryotes that aids in nonhomologous end joining (Aravind and Koonin 2001). Ku binds directly to dsDNA termini and is proven to reduce degradation by $\mathrm{Adn} A B$, a helicase/nuclease in mycobacteria (Sinha et al., 2009). Ku has been used recently in E. coli CFEs to protect LETs and improved transcription significantly, but those yields were only about a third of those transcribed when using GamS or Chi DNA oligos as inhibitors (Yim et al., 2020). However, because of Ku's different mechanism for LET protection, it was proven to be the most effective in lysates made from diverse bacteria. In $B$. subtilis and C. glutamicum extracts, Ku-protected LETs improved transcription 4.43 - and 1.58-fold, respectively, compared to the no inhibitor control (Yim et al., 2020). This is notable improvement, considering GamS and Chi DNA addition to C. glutamicum CFEs actually reduced RNA yield slightly. While Chi DNA enhanced transcription from LETs in B. subtilis CFEs, Ku was about twice as effective.

\section{Linear Expression Template Modifications and Enhanced Design}

Chemical modification of LETs can also reduce their enzymatic degradation in CFEs (Figure 2C). In E. coli, methylation of genomic DNA provides some protection against restriction endonucleases (Marinus and Løbner-Olesen 2014). PCRgenerated LETs, by virtue of being synthesized in vitro, are unmethylated. Post-synthesis methylation via commercially available enzymes was thus tested as an avenue for extra protection against degradation of LETs. LET methylation via dam methyltransferase (adenosine-specific) increased protein yield by $32 \%$ while methylation by $\mathrm{CpG}$ methyltransferase (cytosine-specific) actually lowered protein yield by $18 \%$ (Zhu et al., 2020). This decrease could be because CpG methyltransferase is a eukaryotic enzyme, yielding methylation patterns that might still appear foreign in E. coli. This hypothesis would suggest that it's not simply the presence of methyl groups added by dam methyltransferase that led to increased yield, but the prokaryotic methylation pattern on the LET disguising it as native DNA. The potential number of methyl groups that can be incorporated throughout the LET is restricted with this method, since the commercial methyltransferases act only at sequencespecific sites. These sites can easily be added at regions flanking the gene, but the concentration of methylation sites within the gene itself will be fixed.
Noncanonical DNA backbones and nucleotides can also be used to decrease LET susceptibility to degradation by lysate nucleases. Phosphorothioate (PT) linkages have been shown to restrict exonuclease digestion in vivo, specifically from Lambda exonuclease (Exo) (Putney et al., 1981; Mosberg et al., 2012). Terminal PT linkages can easily be added to LETs during PCR with modified primers. However, these DNA modifications have generally shown insignificant stabilization of LETs in CFEs (Zhu et al., 2020), with the exception of one instance where two PT linkages were added immediately upstream of the $\sigma^{70}$ promoter (Sun et al., 2013). Even still, these PT linkages only improved LET expression by about 36\%. Internal PT linkages might be more effective in protecting dsDNA from Lambda Exo degradation in vivo, as suggested by increased recombination frequency (Mosberg et al., 2012). In addition, several other chemical modifications can be made to LETs via PCR primers that might reduce their susceptibility to degradation, including carbon spacers and 2'-O-methoxy-ethyl bases. However, to our knowledge, these modifications have yet to be tested in CFEs.

Different PCR-based techniques for template DNA production have also been explored for generating templates that provide the highest protein yield while still avoiding the lengthy cloning steps required to make plasmids. With appropriately designed primers, PCR amplicons can be purified and ligated to create circularized templates that give protein yields comparable to plasmid DNA without cloning and transformation (Wu et al., 2007). Furthermore, dilute samples of these recircularized PCR amplicons can be amplified overnight via rolling circle amplification and used directly in CFEs at more suitable concentrations (Dopp et al., 2019). These approaches maintain shorter production times, which is desirable for highthroughput testing, but have not gained much popularity in other applications. Also, their use has been restricted to only T7 expression elements.

Finally, designing the LET sequence in ways that specifically prolong the half-life of the messenger RNA (mRNA) transcripts can significantly improve LET-based production. Increased mRNA half-life is essential for LET yield since it allows for continued protein production even after the LET has been compromised. Use of extracts from strains such as BL21 Star-which has reduced RNase activity due to a mutation in the RNase E gene-is one way to accomplish this. Additionally, the inclusion of various $3^{\prime}$-tail mRNA secondary structures can improve protein yield from LETs, with poly $(\mathrm{G})$ tails and T7 terminator sequences increasing yield by nearly 2 -fold and 3 -fold, respectively (Ahn et al., 2005).

\section{Reduced Culture Temperatures}

It is worth noting that simple modifications to the lysate preparation protocol can sometimes improve LET protein yield, a particularly desirable approach based on its simplicity. It has previously been reported that $E$. coli cultivation temperature can have a significant influence on the exonuclease activity of the lysate. Reducing culture temperature to $30^{\circ} \mathrm{C}$ was shown to approximately triple LETdriven protein yield compared to lysates cultured at $37^{\circ} \mathrm{C}$, while plasmid-driven protein yield decreased slightly (Seki et al., 2008). 
However, others have reported no increased expression from LETs when using this method (Sun et al., 2013).

\section{APPLICATIONS OF LINEAR EXPRESSION TEMPLATES}

While the discussion in Approaches for Stabilizing Linear DNA in Cell-Free Expression Systems often used protein yields as a metric to indicate the relative utility of methods to stabilize LETs, producing large quantities of protein is not the most compelling reason to use LETs. In fact, all of the approaches described above still led to protein yields from LETs below those from plasmids. When producing large amounts of protein is the goal, the use of plasmids is preferred not only due to the attendant improved expression, but also due to the relatively low cost of plasmid production. The most prominent benefits of LETs lie in their ability to accelerate synthetic biology prototyping and enable the expression of genes that would otherwise be toxic to produce in vivo.

\section{Circuit Prototyping}

Rapid prototyping of genetic circuits has been significantly impacted by the use of LETs in CFEs. Genetic circuit design to date has largely been guided by trial-and-error testing, known in the field as the Design-Build-Test-Learn (DBTL) cycle. The length of the DBTL cycle can be substantially shortened by using LETs to avoid time-consuming cloning steps after every iteration. For example, LETs have been used to assemble feedforward loop circuits and quickly facilitate promoter optimization (Guo and Murray 2019). Other complex systems, such as synthetic genetic oscillators (Niederholtmeyer et al., 2015; Yelleswarapu et al., 2018) and four-piece genetic switches have also been be assembled with LETs (Sun et al., 2013). To quantify rates of transcription and translation in real time, fluorescent reporters can be used at the RNA and/or protein level and measured directly. One example of such an assay, PERSIA, uses LETs in CFEs to rapidly characterize several biological phenomena during the reaction (Wick et al., 2019). The activities of T7 promoter mutants have been efficiently characterized through the use of LETs by using the PCR products from an in vitro transcription screen directly in CFEs (Komura et al., 2018). Similarly, using a common reverse primer and unique forward primers, a LET library was quickly generated to test the spatial dependence of promoter and operator sequences at low cost in lysate-based CFEs (McManus et al., 2019).

\section{Engineering Synthetic Regulators}

LETs can also expedite the lengthy screening time required for engineering synthetic regulators. RNA toehold switches are powerful riboregulators that have been used to detect nucleic acid sequences in vivo (Green et al., 2014) and in CFEs, including low-cost user-friendly diagnostics to detect pathogens (Pardee et al., 2016). A toehold-based sensing system consists of two pieces: the RNA toehold switch sequence responsible for translational regulation, and the trans-acting nucleic acid trigger sequence that binds to the switch and modulates its activity. The switch and/or the RNA trigger can be encoded on LETs and expressed in the cell-free reaction (Takahashi et al., 2018), which is particularly important because finding a switch with good performance characteristics (leakiness, limits of detection, etc.) requires screening multiple (and sometimes many) candidates. Also, triggers can be added directly as linear ssDNA oligos to quickly test for switch functionality for a given target, avoiding the encoding of RNA triggers on DNA templates entirely (Amalfitano et al., 2021).

While the above and many other applications of toehold switches have used (nuclease-free) PURE systems, LET-based toehold systems can also be used in crude cell lysate systems for a more cost-efficient way to screen switches. Using Chi DNA as a nuclease inhibitor, ssDNA trigger oligos were stabilized and detected with toehold switches in encapsulated CFEs (Garamella et al., 2019). RNA triggers encoded onto LETs were used in developing a novel crude lysate-based multiplexed diagnostic sensor (Zhang et al., 2021). Additionally, in the more general class of synthetic riboregulators, riboswitch development also suffers from inefficient trial-and-error design (Etzel and Mörl 2017) that can be addressed with LETs in CFEs. This approach has previously been used as a platform to model riboregulator kinetics and predict in vivo behavior (Senoussi et al., 2018).

\section{CRISPR-Cas Screening}

Development and screening of CRISPR systems has also benefited from the rapid prototyping enabled by LETs in CFEs. CRISPR technologies harness the nuclease activities from what is essentially a bacterial "immune system" to execute functions ranging from precise and efficient DNA editing to indiscriminate cleavage of nucleic acid sequences. These functions have been used in a variety of applications from plant and animal genomic manipulation to therapeutics and diagnostics (Barrangou and Doudna 2016; Petri and Pattanayak 2018). However, the laborious process of screening protein function has imposed obstacles to how rapidly advances can be made. This bottleneck has been mitigated in part through the use of CFEs for rapid CRISPR-Cas characterization (Marshall et al., 2018). LETs amplified from genomic fragments containing multiple Cas proteins have been used in CFEs to discover CRISPRCas12a inhibitors (Watters et al., 2018). LETs encoding dozens of guide RNA switch candidates (guide RNAs coupled to RNA toehold switches) were used for rapid characterization in CFEs to develop a modular design scheme for regulating Cas12a activity with arbitrary RNA inducers (Collins et al., 2021). LETs have also been used to elucidate the protospacer-adjacent motif (PAM) sequences recognized by Cas nucleases (Maxwell et al., 2018). While LETs can be used to express a Cas protein as well as its guide RNA sequence, it is particularly advantageous to use LETs for Cas expression since certain Cas genes can be extremely difficult to clone into plasmid vectors containing promoters suitable for CFEs (Maxwell et al., 2018).

\section{Expression of Toxic Proteins}

Beyond Cas, the expression of many metabolically taxing or toxic proteins has been facilitated by LETs. In vivo, some proteins can interfere with metabolic pathways or inhibit cell division, 
resulting in cell death. Since CFEs do not have these same requirements as cells, they are a promising system for expression of such proteins. However, use of plasmids as expression templates in CFEs still requires traditional plasmid cloning, which can be challenging for such toxic proteins. LETs eliminate this challenge, as they can be generated by PCR from a DNA template that is much easier to clone: a plasmid with a defective promoter. For example, the human G-protein coupled receptor (GPCR) has been expressed at cytotoxic levels in both lysate-based CFEs and recombinant protein systems using LETs (Haberstock et al., 2012). Antimicrobial peptides (AMPs), which act as a natural infection defense mechanism and are known to severely stunt bacterial growth, have been successfully expressed with CFEs using LETs (Dopp et al., 2019). Other classes of difficult-to-express proteins that have been expressed successfully off plasmids in CFEs include vaccine antigens (Welsh et al., 2012) and antibiotic efflux pumps (Wuu and Swartz 2008). Employing LETs in these applications could simplify template production or translation to other proteins in these classes.

\section{Other Applications}

LETs also allow CFEs to produce larger protein libraries more easily. Protein microarrays are systems that screen the activity and interaction of many proteins in parallel, but their use is limited by persisting technical challenges including lengthy protein expression, purification, and immobilization steps that reduce the maximum diversity of proteins for array construction (Schinn et al., 2016). The same challenges do not arise with DNA arrays; thus, in situ protein arrays that can use nucleic acid arrays as a template for protein production are advantageous (He et al., 2008b). Two methods that use LETs and CFEs to create protein microarrays include the protein in situ array, PISA (He and Taussig 2001), and a DNA array to protein array approach, DAPA (He et al., 2008a). Both methods involve parallel in situ protein expression and surface capture using purification tags that can be easily added to LETs with PCR. With PISA, $35 \mathrm{fg}$ of unpurified LET was sufficient for detectable production of GFP in sub-nL volumes (Angenendt et al., 2006; Berrade et al., 2011).

LETs have also been shown to be functional in various nonstandard reaction environments for CFEs. Using Chi DNA as a nuclease inhibitor, LETs have been used in polymer microgels to synthesize functional malonyl-CoA synthetase (MatB) (Köhler et al., 2020). LETs have also been spotted on glass slides and aligned to microfluidic devices enclosing each LET into a reaction chamber (Gerber et al., 2009). Microfluidic devices have also been used to encapsulate LET-containing CFEs (with GamS inhibitor) into agarose hydrogel beads, which then withstood lyophilization, storage, and rehydration into a functional sensor for $P$. aeruginosa quorum-sensing molecules (Seto 2019). Moreover, with sufficient protection, LETs can be used in continuous reaction conditions as well. While batch operation is the simplest mode for CFEs, depletion and degradation of reagents can limit long-term productivity. One notable example of a continuous format to address this issue was the use of a multilayer microfluidic device engineered to conduct extended cell-free reactions, where LETs were used to create a screening process that was entirely in vitro (Niederholtmeyer et al., 2013).

\section{THE FUTURE OF LINEAR DNA IN CELL-FREE EXPRESSION SYSTEMS}

Advances in nuclease inhibition strategies to facilitate LET-based expression have made the applications described above, and many others, possible. While existing nuclease inhibition methods are sufficient for certain applications, there is still both room and a critical need for improvement. Further enhancement of linear DNA stability and protein yield from LETs in CFEs can drive innovation of novel synthetic biology research tools and broaden the scope of viable applications of CFEs.

\section{Improvements in Linear Expression Template-Based Expression}

One insufficiently studied aspect of CFEs is the interplay between promoter strength and template DNA structure. Since a common goal for using LETs in CFEs is maximizing protein yield, and since a common concern with using LETs is diminished expression, most scientists designing LETs choose to use strong promoters. As a result, there is little literature characterization of the expression levels from different promoters from LETs. However, use of diverse promoters is often an integral part of engineering genetic circuits and cellfree technologies, meaning that an accurate characterization of promoter strength in LETs is important. Multiple investigations of synthetic $\sigma^{70}$ constitutive promoters on LETs showed that the relative strengths seen in CFEs have no correlation to the strengths seen when using the same promoters on plasmids in CFEs or in vivo (Chappell et al., 2013; Sun et al., 2013).

One hypothesis is that this discrepancy is due to the relationship between transcriptional rate and DNA supercoiling. This is supported by experiments that show stronger correlations of different ribosome binding site (RBS) strengths between linear and plasmid templates, as RBS sequence and resulting mRNA structure are independent of DNA template conformation (Chappell et al., 2013). Others have also explored the effect of supercoiled linear DNA on transcription by testing two different genes in convergent, divergent, and tandem orientations on a single LET (Yeung et al., 2017). Transcription from each configuration was significantly different, most likely because less supercoiling occurs at free DNA ends compared to the center. Recently, a study that focused on the impact of LET length on performance in CFEs verified via atomic force microscopy (AFM) a significant increase in DNA supercoiling as the LET length increased (Nishio et al., 2021). This study also showed that increasing the LET length from 2.8 to $25.7 \mathrm{kbp}$ improved cell-free expression approximately 3-fold, which could be attributed to the positive influence of DNA supercoiling. The benefits of extending LET length have been previously reported while supplementing with GamS, albeit on a 
much smaller scale, where a 2.4-fold increase in LET expression was observed after the addition of just five bp on each end (Sun et al., 2013). Nonetheless, DNA supercoiling, the significance of gene orientation, and LET length should be taken into consideration for optimal design of future LET-based technologies.

It is also important to identify whether linear DNA stabilization techniques are generalizable across lysates from different species of bacteria. E. coli extracts make up the majority of CFEs because their lysate is the simplest to prepare and provides high protein yields, and because E. coli is otherwise a workhorse model organism. However, E. coli extracts are not practical for every application, as they cannot support some functionalities easily (e.g., glycosylation). As a result, the use of other chassis organisms as lysate sources is growing, and thus so will the demand for expression from LETs in CFEs made from those lysates. Some stabilization techniques that are shown to be most effective in E. coli systems (e.g., GamS) are practically ineffective in other CFEs such as $V$. natriegens (Wiegand et al., 2019). This is perhaps to be expected, as different organisms can possess different nucleases. One example of a nuclease inhibition approach with potential to be effective in CFEs of different species would be the small molecule RecBCD inhibitors mentioned in Nuclease Inhibition, as they have been shown to also inhibit AddAB (a helicase/nuclease native to many bacteria) (Amundsen et al., 2012). Interestingly, Chi DNA sites have also been identified in other bacteria, such as B. subtilis and L. lactis, indicating that Chi DNA oligos could potentially be useful in extracts made from these bacteria (Chedin and Kowalczykowski 2002; Marshall et al., 2017). Chi DNA inhibition has recently been tested in B. subtilis CFEs, and only improved LET yield about 2 -fold (Yim et al., 2020). At any rate, identifying which techniques are generalizable-and for those that are not generalizable, identifying alternatives or replacements as needed-will be important in moving this aspect of CFEs forward.

However, it may in fact turn out that lysate-based CFEs from some non-model organisms could actually improve the efficiency of LET-based expression. Chinese hamster ovary $(\mathrm{CHO})$ cell extracts have been shown to allow for sufficient LET functionality to be used for production of "difficult-to-express" proteins (Thoring et al., 2016). Wheat germ CFEs are known to possess undetectable nuclease activity compared to other cell-free systems (Endo and Sawasaki 2003). Surprisingly, in yeast CFEs, expression from LETs has been shown to yield $40-60 \%$ more protein compared to a plasmid DNA template (Gan and Jewett 2014). These LETs included a $\Omega$ leader sequence for initiation, a T7 promoter, and a 3' poly $(\mathrm{A})_{50}$ tail. Lysates from both $S$. frugiperda (Sachse et al., 2013), and P. putida (H. Wang et al., 2018) allow efficient expression from LETs, with the latter allowing for LET-based expression at about $70 \%$ of plasmidbased yields in the absence of any nuclease inhibitors or DNA protection. However, the economic viability for any downstream applications of CFEs based on these other chassis organisms remains to be seen.
Simultaneous inhibition of multiple nucleases could have a substantial impact on linear DNA stability in CFEs. The majority of approaches to nuclease inhibition have focused on RecBCD as the primary DNA nuclease, but significant activity from other nucleases has been recognized yet typically overlooked (Sun et al., 2013). For example, ExoVII has been identified as a key dsDNA and ssDNA nuclease; its removal has improved the preservation of mutations on dsDNA termini in vivo (Mosberg et al., 2012). Fortunately, some of the widely used nuclease inhibitors can inhibit more than one nuclease. For example, GamS can also inhibit SbcCD, another endo/exonuclease in E. coli (Kulkarni and Stahl 1989). Other significant native E. coli nucleases include RecJ, ExoI, and ExoX (Mosberg et al., 2012). Generalized inhibition methods would further improve linear DNA stability and help bring LET-based gene expression closer to plasmid yields.

\section{Linear Expression Templates to Avoid Confounding Effects in Cell-Free Expression Systems}

LETs also have the potential to solve a unique phenomenon sometimes seen in CFEs but infrequently reported. Our group has observed multiple instances where the addition of plasmids expressing unrelated proteins, or even with no gene insert ("empty vectors"), seems to increase protein production from a separate reporter plasmid (Miguez et al., 2021). This is perhaps counterintuitive, as one might expect transcription or translation to be limited by competition for resources, such that adding more plasmids would increase competition for expression resources and thus likely decrease expression from the original plasmid. We hypothesize that this effect could be caused (in certain expression regimes) by RNase competition. Expression from additional plasmids added to the system would increase the total concentration of RNA, which would increase "competition" for RNases and thus in turn yield increased half-life of mRNA transcripts. Even empty vectors contribute to increasing the total RNA concentration in the reaction due to expression from their selectable marker cassettes. Since LETs encode only the gene of interest and no selectable markers, different LETs could be added for each gene to be expressed without the confounding effects typically caused by plasmids.

\section{Aptamers as Sensing Tools in Cell-Free Expression Systems}

In synthetic biology, the engineering of novel genetic circuits is limited most significantly by the characteristics and diversity of extant cellular machinery (Purnick and Weiss 2009). Specifically, in the development of biosensors using CFEs, limitations on the "biological parts" available in nature has led to increasing demand for de novo regulators and synthetic circuit elements with low crosstalk and high sensitivity (Green et al., 2014). The most prominent examples of success in this space allow for rapid and specific detection of arbitrary nucleic acid sequences, which is useful in developing sensors for pathogens and other microbes. Detection of small molecules, however, has been more 


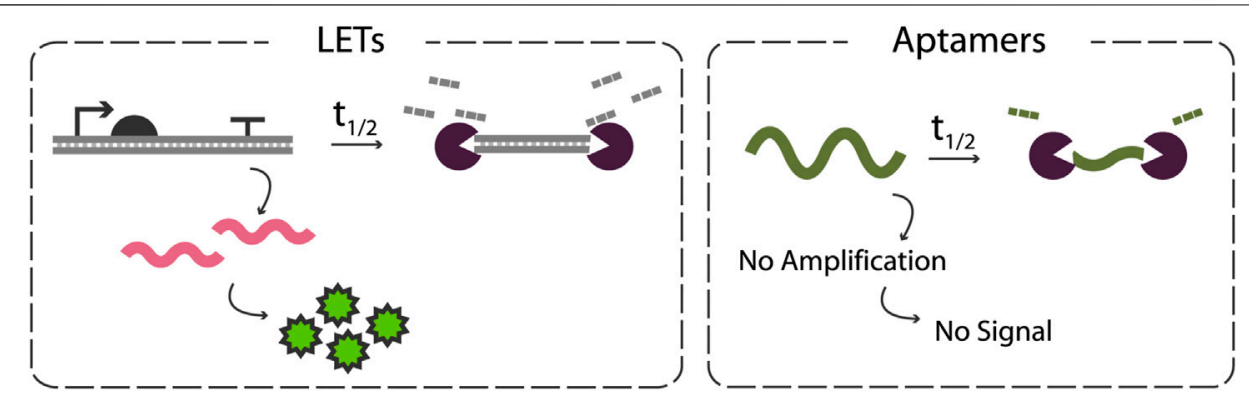

FIGURE 3 | Comparison of the impacts of degradation on LETs vs DNA aptamers in CFEs. LETs benefit from downstream amplification steps, leading to continued protein synthesis even after complete LET degradation. Aptamers, however, must be present and intact to execute their function as they do not serve as templates for amplification.

challenging because there are no de novo-designable regulators to facilitate detection of arbitrary small molecules (Silverman et al., 2020). There is thus a critical need for analogous technologies that can be used in synthetic circuits to detect classes of molecules beyond nucleic acid sequences, including small molecules, proteins, and ions.

Aptamers could potentially fill this need. Aptamers are short oligonucleotides that bind to a specific target with high affinity. DNA aptamers are stable at room temperature, can be synthesized at low cost with negligible batch-to-batch variability, and can withstand lyophilization (Zhang et al., 2019). They have previously been used as the basis for robust sensors for the detection of all types of molecular targets. Most importantly, aptamers are evolved in vitro, which means new aptamers can be created for virtually any analyte.

Despite all of the strengths of DNA aptamers, there are surprisingly few examples of their use in CFEs, likely due to the instability of linear DNA in bacterial extracts. As discussed in Approaches for Stabilizing Linear DNA in Cell-Free Expression Systems, even the best methods for stabilizing LETs still allow significant degradation. DNA aptamers, unlike LETs, are mostly ssDNA oligos, which likely increases their susceptibility to enzymatic degradation in E. coli since there are more native exonucleases that act on ssDNA specifically than there are that act solely on dsDNA (Lovett 2011). Also, aptamers are significantly shorter in length than LETs: LETs include entire coding sequences and other transcription elements, while evolved aptamers are normally 20-60 nucleotides long (Lakhin et al., 2013). Because we know that nucleases can cleave DNA at rates as high as $500 \mathrm{bp} / \mathrm{s}$ (Spies et al., 2007) and that extending LETs results in improved protein yield (Sun et al., 2013), it is likely that the short lengths of aptamers are a major contributor to their instability. This seems reasonable because on a mass basis, aptamers have more free ends than LETs for exonucleases to bind to, and on a molar basis they may have a higher density of critical sequence without which they cannot function.

Another characteristic that sets aptamers apart from LETs is that aptamers do not undergo downstream amplification steps like LETs do (Figure 3). LETs produce numerous mRNA transcripts which are then amplified into protein. These transcripts remain viable templates for protein translation even after the LET has been degraded, and the proteins themselves may also have some function that serves as an amplification of signal in certain applications. Aptamers, however, are not templates for amplification and thus leave no residual function after enzymatic degradation. This means that for aptamers to be used successfully in lysate-based CFEs, nuclease inhibition strategies must be strong enough to prevent aptamer degradation for the entirety of the reaction.

In a few cases, these obstacles have been overcome or circumvented sufficiently to allow DNA aptamers to be successfully used in CFEs. For example, DNA aptamers integrated into plasmids have successfully allowed transcriptional regulation in CFEs (Iyer and Doktycz 2014; Wang et al., 2017). This confirms that the environment of CFEs (in these examples, a commercially available extract) can accommodate proper aptamer secondary structure formation to allow for both target analyte binding and subsequent conformational changes. Integrating the aptamer onto a circular template was done to mitigate exonuclease activity that would otherwise degrade the aptamer. To that end, these reports do not indicate the use of any additional nuclease inhibitors in their experiments.

However, the integration of aptamers onto circular templates can be significantly more labor-intensive and challenging than most of the LET stabilization techniques described above. Since aptamers are single-stranded, incorporation into a circular template typically requires generation of a ssDNA circular template by affinity purification of biotin-labeled ssDNA produced via PCR (Mitchell and Merril 1989). This approach has the advantage of in vitro production but often produces insufficient yields for downstream applications. An alternative method that can produce a higher yield of ssDNA entails in vivo amplification in E. coli with phagemids, which are plasmids that possess both bacteriophage and plasmid properties and thus have all the viral components necessary to enable ssDNA replication (Kuczek et al., 1998; Zhou et al., 2009). However, this approach is even more complex than the in vitro strategy. Regardless of the synthesis strategy, constraining DNA aptamers to circular templates limits the design space and could compromise the functionality of some aptamers. Given all of these considerations, the development of simpler, more effective, and more 
generalizable methods to enable their stability are a critical need to unleash the potential of DNA aptamers in CFEs.

\section{CONCLUSION}

The advances made in nuclease inhibition (specifically of RecBCD) have improved linear DNA stability in lysate-based CFEs sufficiently to allow feasible LET-based production of proteins. The accessibility and ease of implementation of these methods has supported their effective use in driving innovative research in genetic circuit design, efficient screening of de novo riboregulators, rapid screening of CRISPR-Cas system parts, and more. However, continued progress is necessary, because while protein yields from LETs have improved significantly, this is not a direct measurement of LET half-life and obscures the difficulties in using other types of linear DNA constructs in CFEs. Keeping linear DNA stable long enough to function on its own, rather than as a template for amplification, is an important challenge to tackle. Some of the potentially most promising applications of linear DNA in CFEs remain infeasible due to nuclease-based

\section{REFERENCES}

Ahn, J.-H., Chu, H.-S., Kim, T.-W., Oh, I.-S., Choi, C.-Y., Hahn, G.-H., et al. (2005). Cell-Free Synthesis of Recombinant Proteins from PCR-Amplified Genes at a Comparable Productivity to that of Plasmid-Based Reactions. Biochem. Biophysical Res. Commun. 338 (3), 1346-1352. doi:10.1016/j.bbrc.2005.10.094

Amalfitano, E., Karlikow, M., Norouzi, M., Jaenes, K., Cicek, S., Masum, F., et al. (2021). A Glucose Meter Interface for Point-of-Care Gene Circuit-Based Diagnostics. Nat. Commun. 12 (1), 724. doi:10.1038/s41467-020-20639-6

Amundsen, S. K., Spicer, T., Karabulut, A. C., Londoño, L. M., Eberhart, C., Fernandez Vega, V., et al. (2012). Small-Molecule Inhibitors of Bacterial AddAB and RecBCD Helicase-Nuclease DNA Repair Enzymes. ACS Chem. Biol. 7 (5), 879-891. doi:10.1021/cb300018x

Angenendt, P., Kreutzberger, J., Glökler, J., and Hoheisel, J. D. (2006). Generation of High Density Protein Microarrays by Cell-free In Situ Expression of Unpurified PCR Products. Mol. Cell Proteomics 5 (9), 1658-1666. doi:10.1074/mcp.T600024-MCP200

Aravind, L., and Koonin, E. V. (2001). Prokaryotic Homologs of the Eukaryotic DNA-End-Binding Protein Ku, Novel Domains in the Ku Protein and Prediction of a Prokaryotic Double-Strand Break Repair System. Genome Res. 11 (8), 1365-1374. doi:10.1101/gr.181001

Barrangou, R., and Doudna, J. A. (2016). Applications of CRISPR Technologies in Research and beyond. Nat. Biotechnol. 34 (9), 933-941. doi:10.1038/nbt.3659

Berrade, L., Garcia, A. E., and Camarero, J. A. (2011). Protein Microarrays: Novel Developments and Applications. Pharm. Res. 28 (7), 1480-1499. doi:10.1007/ s11095-010-0325-1

Chappell, J., Jensen, K., and Freemont, P. S. (2013). Validation of an Entirely In Vitro Approach for Rapid Prototyping of DNA Regulatory Elements for Synthetic Biology. Nucleic Acids Res. 41 (5), 3471-3481. doi:10.1093/nar/gkt052

Chedin, F., and Kowalczykowski, S. C. (2002). A Novel Family of Regulated Helicases/Nucleases from Gram-Positive Bacteria: Insights into the Initiation of DNA Recombination. Mol. Microbiol. 43 (4), 823-834. doi:10.1046/j.13652958.2002.02785.x

Collins, S. P., Rostain, W., Liao, C., and Beisel, C. L. (2021). Sequence-independent RNA Sensing and DNA Targeting by a Split Domain CRISPR-Cas12a gRNA Switch. Nucleic Acids Res. 49, 2985-2999. doi:10.1093/nar/gkab100

Contreras-Llano, L. E., Meyer, C., Liu, Y., Sarker, M., Lim, S., Longo, M. L., et al. (2020). Holistic Engineering of Cell-free Systems through ProteomeReprogramming Synthetic Circuits. Nat. Commun. 11 (1), 3138. doi:10.1038/s41467-020-16900-7 degradation, meaning that more effective methods to extend linear DNA half-life could have a dramatic, enabling impact on a myriad of biotechnology applications.

\section{AUTHOR CONTRIBUTIONS}

Writing-original draft: MM. Writing-review and editing: MS.

\section{FUNDING}

The authors thank the National Institutes of Health (R01EB022592) and the National Science Foundation (CCF2007807) for funding support.

\section{ACKNOWLEDGMENTS}

The authors thank Alexandra Patterson for insightful discussions regarding DNA methylation.

Dopp, J. L., Rothstein, S. M., Mansell, T. J., and Reuel, N. F. (2019). Rapid Prototyping of Proteins: Mail Order Gene Fragments to Assayable Proteins within 24 hours. Biotechnol. Bioeng. 116 (3), 667-676. doi:10.1002/ bit.26912

Endo, Y., and Sawasaki, T. (2003). High-Throughput, Genome-Scale Protein Production Method Based on the Wheat Germ Cell-free Expression System. Biotechnol. Adv. 21 (8), 695-713. doi:10.1016/S0734-9750(03)00105-8

Etzel, M., and Mörl, M. (2017). Synthetic Riboswitches: From Plug and Pray toward Plug and Play. Biochemistry 56 (9), 1181-1198. doi:10.1021/ acs.biochem.6b01218

Gan, R., and Jewett, M. C. (2014). A Combined Cell-Free TranscriptionTranslation System from Saccharomyces Cerevisiaefor Rapid and Robust Protein Synthesis. Biotechnol. J. 9 (5), 641-651. doi:10.1002/biot.201300545

Garamella, J., Majumder, S., Liu, A. P., and Noireaux, V. (2019). An Adaptive Synthetic Cell Based on Mechanosensing, Biosensing, and Inducible Gene Circuits. ACS Synth. Biol. 8 (8), 1913-1920. doi:10.1021/acssynbio.9b00204

Gerber, D., Maerkl, S. J., and Quake, S. R. (2009). An In Vitro Microfluidic Approach to Generating Protein-Interaction Networks. Nat. Methods 6 (1), 71-74. doi:10.1038/nmeth.1289

Green, A. A., Silver, P. A. James. J. Collins., Collins, J. J., and Yin, P. (2014). Toehold Switches: De-novo-designed Regulators of Gene Expression. Cell 159 (4), 925-939. doi:10.1016/j.cell.2014.10.002

Guo, S., and Murray, R. M. (2019). Construction of Incoherent Feedforward Loop Circuits in a Cell-free System and in Cells. ACS Synth. Biol. 8 (3), 606-610. doi:10.1021/acssynbio.8b00493

Haberstock, S., Roos, C., Hoevels, Y., Dötsch, V., Schnapp, G., Pautsch, A., et al. (2012). A Systematic Approach to Increase the Efficiency of Membrane Protein Production in Cell-free Expression Systems. Protein Expr. Purif. 82 (2), 308-316. doi:10.1016/j.pep.2012.01.018

He, M., Stoevesandt, O., Palmer, E. A., Khan, F., Ericsson, O., Taussig, M. J., et al. (2008a). Printing Protein Arrays from DNA Arrays. Nat. Methods 5 (2), 175-177. doi:10.1038/nmeth.1178

He, M., Stoevesandt, O., and Taussig, M. J. (2008b). In Situ Synthesis of Protein Arrays. Curr. Opin. Biotechnol. 19 (1), 4-9. doi:10.1016/j.copbio. 2007.11.009

He, M., and Taussig, Michael. J. (2001). Single Step Generation of Protein Arrays from DNA by Cell-free Expression and In Situ Immobilisation (PISA Method). Nucleic Acids Res. 29 (15), 73e-73. doi:10.1093/nar/29.15.e73

Iyer, S., and Doktycz, M. J. (2014). Thrombin-Mediated Transcriptional Regulation Using DNA Aptamers in DNA-Based Cell-free Protein Synthesis. ACS Synth. Biol. 3 (6), 340-346. doi:10.1021/sb4000756 
Jana, R., Hazbun, T. R., Fields, J. D., and Mossing, M. C. (1998). Single-Chain Lambda Cro Repressors Confirm High Intrinsic Dimer-DNA Affinity†. Biochemistry 37 (18), 6446-6455. doi:10.1021/bi980152v

Katzen, F., Chang, G., and Kudlicki, W. (2005). The Past, Present and Future of Cell-free Protein Synthesis. Trends Biotechnol. 23 (3), 150-156. doi:10.1016/ j.tibtech.2005.01.003

Khambhati, K., Bhattacharjee, G., Gohil, N., Braddick, D., Kulkarni, V., and Singh, V. (2019). Exploring the Potential of Cell-free Protein Synthesis for Extending the Abilities of Biological Systems. Front. Bioeng. Biotechnol. 7. doi:10.3389/ fbioe.2019.00248

Köhler, T., Heida, T., Hoefgen, S., Weigel, N., Valiante, V., and Thiele, J. (2020). Cell-Free Protein Synthesis and In Situ Immobilization of DeGFP-MatB in Polymer Microgels for Malonate-To-Malonyl CoA Conversion. RSC Adv. 10 (66), 40588-40596. doi:10.1039/D0RA06702D

Komura, R., Aoki, W., Motone, K., Satomura, A., and Ueda, M. (2018). HighThroughput Evaluation of T7 Promoter Variants Using Biased Randomization and DNA Barcoding. PLOS ONE 13 (5), e0196905. doi:10.1371/ journal.pone. 0196905

Kuczek, K., Kotowska, M., Wiernik, D., and Mordarski, M. (1998). Single-Stranded DNA Production from Phagemids Containing GC-Rich DNA Fragments. BioTechniques 24 (2), 214-215. doi:10.2144/98242bm08

Kulkarni, S. K., and Stahl, F. W. (1989). Interaction between the SbcC Gene of Escherichia Coli and the Gam Gene of Phage Lambda. Genetics 123 (2), 249-253. doi:10.1093/genetics/123.2.249

Kuzminov, A., and Stahl, F. W. (1997). Stability of Linear DNA in RecA Mutant Escherichia Coli Cells Reflects Ongoing Chromosomal DNA Degradation. J. Bacteriol. 179 (3), 880-888. doi:10.1128/jb.179.3.880-888.1997

Kwon, Y.-C., and Jewett, M. C. (2015). High-Throughput Preparation Methods of Crude Extract for Robust Cell-free Protein Synthesis. Sci. Rep. 5 (1), 8663. doi:10.1038/srep08663

Lakhin, A. V., Tarantul, V. Z., and Gening, L. V. (2013). Aptamers: Problems, Solutions and Prospects. Acta Naturae 5 (4), 34-43. doi:10.32607/207582512013-5-4-34-43

Laohakunakom, N., Grasemann, L., Lavickova, B., Michielin, G., Shahein, A., Swank, Z., et al. (2020). Bottom-Up Construction of Complex Biomolecular Systems with Cell-free Synthetic Biology. Front Bioeng. Biotechnol. 8, 213. doi:10.3389/fbioe.2020.00213

Lavickova, B., and Maerkl, S. J. (2019). A Simple, Robust, and Low-Cost Method to Produce the PURE Cell-free System. ACS Synth. Biol. 8 (2), 455-462. doi:10.1021/acssynbio. 8 b00427

Lovett, S. T. (2011). The DNA Exonucleases of Escherichia Coli. EcoSal Plus 4 (2). doi:10.1128/ecosalplus.4.4.7

Marinus, M. G., and Løbner-Olesen, A. (2014). DNA Methylation. EcoSal Plus 6 (1). doi:10.1128/ecosalplus.ESP-0003-2013

Marshall, R., Maxwell, C. S., Collins, S. P., Beisel, C. L., and Noireaux, V. (2017). Short DNA Containing $\chi$ Sites Enhances DNA Stability and Gene Expression in E. coli Cell-free Transcription-Translation Systems. Biotechnol. Bioeng. 114 (9), 2137-2141. doi:10.1002/bit.26333

Marshall, R., Maxwell, C. S., Collins, S. P., Jacobsen, T., Luo, M. L., Begemann, M. B., et al. (2018). Rapid and Scalable Characterization of CRISPR Technologies Using an E. Coli Cell-free Transcription-Translation System. Mol. Cell 69 (1), 146-157. doi:10.1016/j.molcel.2017.12.007

Maxwell, C. S., Jacobsen, T., Marshall, R., Noireaux, V., and Beisel, C. L. (2018). A Detailed Cell-free Transcription-Translation-Based Assay to Decipher CRISPR Protospacer-Adjacent Motifs. Methods 143 (July), 48-57. doi:10.1016/ j.ymeth.2018.02.016

McManus, J. B., Emanuel, P. A., Murray, R. M., and Lux, M. W. (2019). A Method for Cost-Effective and Rapid Characterization of Engineered T7-Based Transcription Factors by Cell-free Protein Synthesis Reveals Insights into the Regulation of T7 RNA Polymerase-Driven Expression. Arch. Biochem. Biophys. 674 (October), 108045. doi:10.1016/j.abb.2019.07.010

McNerney, M. P., Zhang, Y. P. S., Steppe, P.A. D., Jewett, M. C., and Styczynski, M. P. (2019). Point-of-Care Biomarker Quantification Enabled by Sample-specific Calibration. Sci. Adv. 5 (9), eaax4473. doi:10.1126/sciadv.aax4473

Michel-Reydellet, N., Woodrow, K., and Swartz, J. (2005). Increasing PCR Fragment Stability and Protein Yields in a Cell-free System with Genetically Modified Escherichia Coli Extracts. J. Mol. Microbiol. Biotechnol. 9 (1), 26-34. doi:10.1159/000088143
Miguez, A. M., Zhang, Y., Piorino, F., and Styczynski, M. P. (2021). Metabolic Dynamics in Escherichia Coli-Based Cell-Free Systems. bioRxiv. doi:10.1101/ 2021.05.16.444339

Mitchell, L. G., and Merril, C. R. (1989). Affinity Generation of Single-Stranded DNA for Dideoxy Sequencing Following the Polymerase Chain Reaction. Anal. Biochem. 178 (2), 239-242. doi:10.1016/0003-2697(89)90631-3

Mosberg, J. A., Gregg, C. J., Lajoie, M. J., Wang, H. H., and Church, G. M. (2012). Improving Lambda Red Genome Engineering in Escherichia Coli via Rational Removal of Endogenous Nucleases. PLOS ONE 7 (9), e44638. doi:10.1371/ journal.pone.0044638

Murphy, K. C. (1991). Lambda Gam Protein Inhibits the Helicase and ChiStimulated Recombination Activities of Escherichia Coli RecBCD Enzyme. J. Bacteriol. 173 (18), 5808-5821. doi:10.1128/jb.173.18.5808-5821.1991

Niederholtmeyer, H., Stepanova, V., and Maerkl, S. J. (2013). Implementation of Cell-free Biological Networks at Steady State. Proc. Natl. Acad. Sci. 110 (40), 15985-15990. doi:10.1073/pnas.1311166110

Niederholtmeyer, H., Sun, Z. Z., Hori, Y., Yeung, E., Verpoorte, A., Murray, R. M., et al. (2015). Rapid Cell-free Forward Engineering of Novel Genetic Ring Oscillators. ELife 4 (October), e09771. doi:10.7554/eLife.09771

Nirenberg, M., and Leder, P. (1964). RNA Codewords and Protein Synthesis: The Effect of Trinucleotides upon the Binding of SRNA to Ribosomes. Science 145 (3639), 1399-1407. doi:10.1126/science.145.3639.1399

Nishio, T., Yoshikawa, Y., Yoshikawa, K., and Sato, S.-i. (2021). Longer DNA Exhibits Greater Potential for Cell-free Gene Expression. Sci. Rep. 11 (1), 11739. doi:10.1038/s41598-021-91243-x

Pardee, K., Green, A. A., Ferrante, T., Cameron, D. E., DaleyKeyser, A., Yin, P., et al. (2014). Green, Tom Ferrante, D. Ewen Cameron, Ajay DaleyKeyser, Peng Yin, andPaper-Based Synthetic Gene Networks. Cell 159 (4), 940-954. doi:10.1016/j.cell.2014.10.004

Pardee, K., Green, A. A., Takahashi, M. K., Braff, D., Lambert, G., Lee, J. W., et al. (2016). Rapid, Low-Cost Detection of Zika Virus Using Programmable Biomolecular Components. Cell 165 (5), 1255-1266. doi:10.1016/ j.cell.2016.04.059

Petri, K., and Pattanayak, V. (2018). SHERLOCK and DETECTR Open a New Frontier in Molecular Diagnostics. CRISPR J. 1 (3), 209-211. doi:10.1089/ crispr.2018.29018.kpe

Purnick, P. E. M., and Weiss., R. (2009). The Second Wave of Synthetic Biology: From Modules to Systems. Nat. Rev. Mol. Cell Biol 10 (6), 410-422. doi:10.1038/ nrm2698

Putney, S. D., Benkovic, S. J., and Schimmel, P. R. (1981). “A DNA Fragment with an Alpha-Phosphorothioate Nucleotide at One End is Asymmetrically Blocked From Digestion by Exonuclease III and can be Replicated In Vivo. Proc. Natl. Acad. Sci. 78 (12), 7350-7354. doi:10.1073/pnas.78.12.7350

Sachse, R., Wüstenhagen, D., Šamalíková, M., Gerrits, M., Bier, F. F., and Kubick, S. (2013). Synthesis of Membrane Proteins in Eukaryotic Cell-free Systems. Eng. Life Sci. 13 (1), 39-48. doi:10.1002/elsc.201100235

Schinn, S.-M., Broadbent, A., Bradley, W. T., and Bundy, B. C. (2016). Protein Synthesis Directly from PCR: Progress and Applications of Cell-free Protein Synthesis with Linear DNA. New Biotechnol. 33 (4), 480-487. doi:10.1016/ j.nbt.2016.04.002

Schoborg, J. A., Hershewe, J. M., Stark, J. C., Kightlinger, W., Kath, J. E., Jaroentomeechai, T., et al. (2018). A Cell-free Platform for Rapid Synthesis and Testing of Active Oligosaccharyltransferases. Biotechnol. Bioeng. 115 (3), 739-750. doi:10.1002/bit.26502

Seki, E., Matsuda, N., and Kigawa, T. (2009). Multiple Inhibitory Factor Removal from an Escherichia Coli Cell Extract Improves Cell-free Protein Synthesis. J. Biosci. Bioeng. 108 (1), 30-35. doi:10.1016/j.jbiosc.2009.02.011

Seki, E., Matsuda, N., Yokoyama, S., and Kigawa, T. (2008). Cell-Free Protein Synthesis System from Escherichia Coli Cells Cultured at Decreased Temperatures Improves Productivity by Decreasing DNA Template Degradation. Anal. Biochem. 377 (2), 156-161. doi:10.1016/j.ab.2008.03.001

Senoussi, A., Lee Tin Wah, J., Shimizu, Y., Robert, J., Jaramillo, A., Findeiss, S., et al. (2018). Quantitative Characterization of Translational Riboregulators Using an In Vitro Transcription-Translation System. ACS Synth. Biol. 7 (5), 1269-1278. doi:10.1021/acssynbio.7b00387

Seto, J. (2019). On a Robust, Sensitive Cell-free Method for Pseudomonas Sensing and Quantification in Microfluidic Templated Hydrogels. Micromachines 10 (8), 506. doi:10.3390/mi10080506 
Shimizu, Y., Inoue, A., Tomari, Y., Suzuki, T., Yokogawa, T., Nishikawa, K., et al. (2001). Cell-Free Translation Reconstituted with Purified Components. Nat. Biotechnol. 19 (8), 751-755. doi:10.1038/90802

Shrestha, P., Smith, M. T., and Bundy, B. C. (2014). Cell-Free Unnatural Amino Acid Incorporation with Alternative Energy Systems and Linear Expression Templates. New Biotechnol. 31 (1), 28-34. doi:10.1016/j.nbt.2013.09.002

Silverman, A. D., Karim, A. S., and Jewett, M. C. (2020). Cell-Free Gene Expression: An Expanded Repertoire of Applications. Nat. Rev. Genet. 21 (3), 151-170. doi:10.1038/s41576-019-0186-3

Sinha, K. M., Unciuleac, M.-C., Glickman, M. S., and Shuman, S. (2009). AdnAB: a New DSB-Resecting Motor-Nuclease from Mycobacteria. Genes Development 23 (12), 1423-1437. doi:10.1101/gad.1805709

Sitaraman, K., Esposito, D., Klarmann, G., Le Grice, S. F., Hartley, J. L., Chatterjee, D. K., et al. (2004). A Novel Cell-free Protein Synthesis System. J. Biotechnol. 110 (3), 257-263. doi:10.1016/j.jbiotec.2004.02.014

Smith, G. R., Kunes, S. M., Schultz, D. W., Taylor, A., and Triman, K. L. (1981). Structure of Chi Hotspots of Generalized Recombination. Cell 24 (2), 429-436. doi:10.1016/0092-8674(81)90333-0

Spies, M., Amitani, I., Baskin, R. J., and Kowalczykowski, S. C. (2007). RecBCD Enzyme Switches Lead Motor Subunits in Response to $\chi$ Recognition. Cell 131 (4), 694-705. doi:10.1016/j.cell.2007.09.023

Sun, Z. Z., Yeung, E., Hayes, C. A., Noireaux, V., and Murray, R. M. (2013). Linear DNA for Rapid Prototyping of Synthetic Biological Circuits in anEscherichia coliBased TX-TL Cell-free System. ACS Synth. Biol. 3, 387-397. doi:10.1021/ sb400131a

Swartz, J. (2006). Developing Cell-free Biology for Industrial Applications. J. Ind. Microbiol. Biotechnol. 33 (7), 476-485. doi:10.1007/s10295-006-0127-y

Takahashi, M. K., Tan, X., Dy, A. J., Braff, D., Akana, R. T., Furuta, Y., et al. (2018). A Low-Cost Paper-Based Synthetic Biology Platform for Analyzing Gut Microbiota and Host Biomarkers. Nat. Commun. 9, 1-12. doi:10.1038/ s41467-018-05864-4

Thavarajah, W., Silverman, A. D., Verosloff, M. S., Kelley-Loughnane, N., Jewett, M. C., and Lucks, J. B. (2020). Point-of-Use Detection of Environmental Fluoride via a Cell-free Riboswitch-Based Biosensor. ACS Synth. Biol. 9 (1), 10-18. doi:10.1021/acssynbio.9b00347

Thoring, L., Wüstenhagen, D. A., Borowiak, M., Stech, M., Sonnabend, A., and Kubick, S. (2016). Cell-Free Systems Based on CHO Cell Lysates: Optimization Strategies, Synthesis of "Difficult-To-Express" Proteins and Future Perspectives. PLOS ONE 11 (9), e0163670. doi:10.1371/journal.pone.0163670

Verosloff, M., Chappell, J., Perry, K. L., Thompson, J. R., and Lucks, J. B. (2019). PLANT-dx: A Molecular Diagnostic for Point-of-Use Detection of Plant Pathogens. ACS Synth. Biol. 8 (4), 902-905. doi:10.1021/acssynbio.8b00526

Wang, H., Li, J., and Jewett, M. C. (2018). Development of a Pseudomonas Putida Cell-free Protein Synthesis Platform for Rapid Screening of Gene Regulatory Elements. Synth. Biol. 3 (1), 1-7. doi:10.1093/synbio/ysy003

Wang, J., Yang, L., Cui, X., Zhang, Z., Dong, L., and Guan, N. (2017). A DNA Bubble-Mediated Gene Regulation System Based on Thrombin-Bound DNA Aptamers. ACS Synth. Biol. 6 (5), 758-765. doi:10.1021/ acssynbio.6b00391

Watters, K. E., Fellmann, C., Bai, H. B, Ren, S. M., and Doudna, J. A. (2018). Systematic Discovery of Natural CRISPR-Cas12a Inhibitors. Science 362 (6411), 236-239. doi:10.1126/science.aau5138

Welsh, J. P., Lu, Y., He, X.-S., Greenberg, H. B., and Swartz, J. R. (2012). Cell-Free Production of Trimeric Influenza Hemagglutinin Head Domain Proteins as Vaccine Antigens. Biotechnol. Bioeng. 109 (12), 2962-2969. doi:10.1002/ bit. 24581

Wick, S., Walsh, D. I., Bobrow, J., Hamad-Schifferli, K., Kong, D. S., Thorsen, T., et al. (2019). PERSIA for Direct Fluorescence Measurements of Transcription,
Translation, and Enzyme Activity in Cell-free Systems. ACS Synth. Biol. 8 (5), 1010-1025. doi:10.1021/acssynbio.8b00450

Wiegand, D. J., Lee, H. H., Ostrov, N., and Church, G. M. (2019). Cell-Free Protein Expression Using the Rapidly Growing Bacterium Vibrio Natriegens. JoVE 145, 1-14. doi:10.3791/59495

Wu, P. S. C., Ozawa, K., Lim, S. P., Vasudevan, S. G., Dixon, N. E., and Otting, G. (2007). Cell-Free Transcription/Translation from PCR-Amplified DNA for High-Throughput NMR Studies. Angew. Chem. Int. Ed. 46 (18), 3356-3358. doi:10.1002/anie.200605237

Wuu, J. J., and Swartz, J. R. (2008). High Yield Cell-free Production of Integral Membrane Proteins without Refolding or Detergents. Biochim. Biophys. Acta (Bba) - Biomembranes 1778 (5), 1237-1250. doi:10.1016/j.bbamem.2008.01.023

Yelleswarapu, M., van der Lindenvan der Linden, A. J., van Sluijs, B., Pieters, P. A., Dubuc, E., de Greef, T. F. A., et al. (2018). Sigma Factor-Mediated Tuning of Bacterial Cell-free Synthetic Genetic Oscillators. ACS Synth. Biol. 7 (12), 2879-2887. doi:10.1021/acssynbio.8b00300

Yeung, E., Dy, A. J., Martin, K. B., Ng, A. H., Del Vecchio, D., Beck, J. L., et al. (2017). Biophysical Constraints Arising from Compositional Context in Synthetic Gene Networks. Cell Syst. 5 (1), 11-24.e12. doi:10.1016/ j.cels.2017.06.001

Yim, S. S., Johns, N. I., Vincent, N., and Wang, H. H. (2020). Protecting Linear DNA Templates in Cell-free Expression Systems from Diverse Bacteria. ACS Synth. Biol. 9 (10), 2851-2855. doi:10.1021/acssynbio.0c00277

Yu, D., Ellis, H. M., Lee, E.-C., Jenkins, N. A., Copeland, N. G., and Court, D. L. (2000). An Efficient Recombination System for Chromosome Engineering in Escherichia Coli. Proc. Natl. Acad. Sci. 97 (11), 5978-5983. doi:10.1073/ pnas. 100127597

Zawada, J. F., Yin, G., Steiner, A. R., Yang, J., Naresh, A., Roy, S. M., et al. (2011). Microscale to Manufacturing Scale-up of Cell-free Cytokine Production-A New Approach for Shortening Protein Production Development Timelines. Biotechnol. Bioeng. 108 (7), 1570-1578. doi:10.1002/bit.23103

Zawada, J., and Swartz, J. (2006). Effects of Growth Rate on Cell Extract Performance in Cell-free Protein Synthesis. Biotechnol. Bioeng. 94 (4), 618-624. doi:10.1002/bit.20831

Zhang, Y., Kojima, T., Kim, G.-A., McNerney, M. P., Takayama, S., and Styczynski, M. P. (2021). Protocell Arrays for Simultaneous Detection of Diverse Analytes. bioRxiv. doi:10.1101/2021.02.13.431022

Zhang, Y., Lai, B., and Juhas, M. (2019). Recent Advances in Aptamer Discovery and Applications. Molecules 24 (5), 941. doi:10.3390/molecules24050941

Zhou, B., Dong, Q., Ma, R., Chen, Y., Yang, J., Sun, L.-Z., et al. (2009). Rapid Isolation of Highly Pure Single-Stranded DNA from Phagemids. Anal. Biochem. 389 (2), 177-179. doi:10.1016/j.ab.2009.03.044

Zhu, B., Gan, R., Cabezas, M. D., Kojima, T., Nicol, R., Jewett, M. C., et al. (2020). Increasing Cell-free Gene Expression Yields from Linear Templates in Escherichia coli and Vibrio Natriegens Extracts by Using DNA-binding Proteins. Biotechnol. Bioeng. 117 (12), 3849-3857. doi:10.1002/bit.27538

Conflict of Interest: The authors declare that the research was conducted in the absence of any commercial or financial relationships that could be construed as a potential conflict of interest.

Copyright (c) 2021 McSweeney and Styczynski. This is an open-access article distributed under the terms of the Creative Commons Attribution License (CC $B Y)$. The use, distribution or reproduction in other forums is permitted, provided the original author(s) and the copyright owner(s) are credited and that the original publication in this journal is cited, in accordance with accepted academic practice. No use, distribution or reproduction is permitted which does not comply with these terms. 